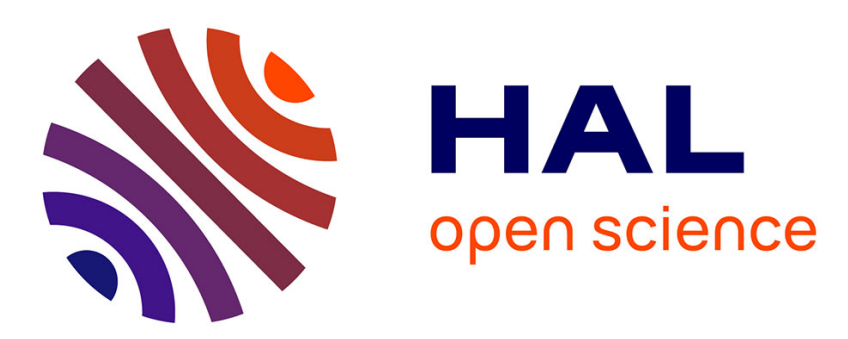

\title{
Robust Control of a Competitive Environment in the Chemostat using Discontinuous Control Laws
}

Alex dos Reis de Souza, Denis Efimov, Andrey Polyakov, Jean-Luc Gouzé

\section{To cite this version:}

Alex dos Reis de Souza, Denis Efimov, Andrey Polyakov, Jean-Luc Gouzé. Robust Control of a Competitive Environment in the Chemostat using Discontinuous Control Laws. CDC 2019 - 58th IEEE Conference on Decision and Control, Dec 2019, Nice, France. hal-02308076

\section{HAL Id: hal-02308076 https://hal.inria.fr/hal-02308076}

Submitted on 8 Oct 2019

HAL is a multi-disciplinary open access archive for the deposit and dissemination of scientific research documents, whether they are published or not. The documents may come from teaching and research institutions in France or abroad, or from public or private research centers.
L'archive ouverte pluridisciplinaire $\mathbf{H A L}$, est destinée au dépôt et à la diffusion de documents scientifiques de niveau recherche, publiés ou non, émanant des établissements d'enseignement et de recherche français ou étrangers, des laboratoires publics ou privés. 


\title{
Robust Control of a Competitive Environment in the Chemostat using Discontinuous Control Laws
}

\author{
Alex dos Reis de Souza, Denis Efimov, Andrey Polyakov and Jean-Luc Gouzé
}

\begin{abstract}
This work addresses the problem of robust stabilization of the concentration of two different species competing for a single limiting substrate. This stabilization is performed by means of discontinuous feedback control laws that ensure coexistence of all species. The control laws are designed considering bounded uncertainties on the kinetic rates.
\end{abstract}

\section{INTRODUCTION}

In modern and practical biotechnological and biochemical applications, bioreactors are widely known for allowing experiments involving living micro-organisms, under controlled conditions and that mimic a natural environment. Many applications arise from such experiments, like pharmaceutical production, yeast fermentation, ethanol production, polymerization and many others. A bioreactor might operate in three distinct modes [1]: batch, fed-batch and continuous. In the first, media is added and the process allowed to proceed until a certain condition is reached, in the second fresh media can be continuously fed into the bioreactor but not removed and, finally, the third comprehends the case in which fresh media might be added and removed proportionally. A well-known example of continuous bioreactor is the chemostat.

The competitive exclusion principle [2] states that the competition between $N$ species for a single limiting substrate results in the extinction (or wash-out) of all but one species. This fact encourages the study of methodologies to overcome such a drawback in laboratory-like environments, since coexistence is observed in nature.

The problem of stabilization of the chemostat was widely studied in the last years. Many approaches for designing control laws with such an objective were discussed. In the literature, there are many interesting works addressing feedback control [3] [4], adaptive control [5] and robust control [6] [7] [8] [9].

Robustness is an important feature when dealing with control systems, since real and practical applications are often plagued by uncertainties, exogenous disturbances or even unmodeled dynamics. In this sense, robust control laws aim to insure stability and performance even in these cases. Specifically about bioprocesses, the main source of uncertainty relates to the kinetic rates [10].

This work was supported by IPL CoSY.

Alex dos Reis de Souza, Denis Efimov, Andrey Polyakov are with Inria - Lille Nord Europe, Université de Lille CNRS, UMR 9189 - CRIStAL, F-59000 Lille, France. E-mail: (alex.dos-reis-de-souza, denis.efimov, andrey.polyakov) dinria.fr

Jean-Luc Gouzé is with Université Côte d'Azur, Inria, INRA, CNRS, Sorbonne Université, Biocore Team, Sophia Antipolis, France. E-mail: jean-luc.gouzedinria.fr
In [8], the authors have introduced the interesting concept of robustness to biodiversity, which discusses the performance of a certain control law in a biological framework with many species, taking into account their competition, selection and coexistence.

Sliding mode control is a discontinuous non-linear control method, being widely known for characteristics such as simplicity of design, finite-times rates of convergence and robustness [11]. This technique has proven its usefulness to a wide range of applications, such as regulation, trajectory tracking and observation. Also, it is worth evoking the work of [12], where it has been reported an evidence of a quasisliding mode controller in a biological context.

In this work, we aim to further discuss about the stabilization of two species in a continuous bioreactor, under competition for a single limiting nutrient. We consider that the kinetic rates, which describe nutrient uptake by each species, are uncertain with respect to their parameters. In this sense, we study the applicability of discontinuous control laws - specifically sliding mode control - that insure the persistence of both species despite of these uncertainties. The work here presented differs from the ones present in the literature for providing robust stabilization even in the competition scenario. Furthermore, the methodology we developed aims to simplify and to drop harsh assumptions such as perfect knowledge on the kinetic rates - that might impose difficulties for future practical implementations.

Structure of the paper: the problem statement is presented in Section 2. Preliminary concepts are introduced in Section 3 and the main results are stated in Section 4. Numerical examples illustrate the intended application in Section 5 and concluding remarks and future directions are discussed in Section 6.

\section{Problem Statement}

Consider the following non-linear system, which describes microbial growth of 2 distinct species inside a chemostat with a single limiting substrate (time-dependence was omitted for readability):

$$
\begin{aligned}
\frac{d S}{d t} & =\left(S_{i n}-S\right) D-\sum_{i=1}^{2} k_{i} \mu_{i}(S) x_{i} \\
\frac{d x_{i}}{d t} & =\left(\mu_{i}(S)-D\right) x_{i} \quad i=1,2 \\
S(0) & >0 \\
x_{i}(0) & >0 \quad i=1,2
\end{aligned}
$$


where $S$ and $x_{i}$ are, respectively, the concentration of the substrate and the $i$-th species, $S_{i n}$ and $D$ are the control inputs (nutrient inflow concentration and dilution rate, respectively), supposed to be limited such as $S_{i n} \in\left[0, S_{i n}^{\max }\right]$ and $D \in\left[0, D_{\max }\right]$. Also, $k_{i}$ is the constant yield coefficient and $\mu_{i}(S)$ is the specific growth rate, describing the nutrient uptake kinetics by the i-th species, given by Monod's law as follows:

$$
\mu_{i}(S)=\frac{a_{i} S}{b_{i}+S}
$$

where $a_{i}$ and $b_{i}$ are supposed to be uncertain, such that $a_{i} \in A_{i}=\left[\underline{a_{i}}, \overline{a_{i}}\right]$ and $b_{i} \in B_{i}=\left[\underline{b_{i}}, \overline{b_{i}}\right]$ for $i=1,2$, where $\underline{a_{i}}, \overline{a_{i}}, \underline{b_{i}}, \overline{b_{i}}$ are known bounds, obtained from experimental procedures in a laboratory. These uncertainties relate to possible fluctuations (possibly time-varying behavior) on the microbial community.

The aim of this paper is to design a robust control methodology, using discontinuous feedback laws, that ensures coexistence of two species despite the aforementioned uncertainties and stabilizes each one in a desired set-point $x_{i}^{d}, i=1,2$. Furthermore, in this initial work, we supposed that measurements of the whole state vector are available.

\section{PRELIMINARIES}

\section{A. Robust exact differentiator}

The design of real-time differentiators is a well-known and studied problem nowadays (see the special issue on differentiators [13]). The main challenge to such an implementation is sensitivity to noise. Many approaches to design differentiators became popular throughout the years, like high-gain observers and sliding-mode differentiators.

In this section, we use results from [14] for the design of an arbitrary-order, robust and exact differentiator. These exact differentiators demonstrate a finite-time convergence and also good sensitivity to input noise.

Let an output signal $f(t)=f_{0}(t)+w(t)$ to be defined in $[0, \infty)$ and being corrupted by an unknown but Lebesguemeasurable noise $w(t)$, where an unknown base signal $f_{0}(t)$ has its $n$-th derivative having a known Lipschitz constant $L>$ 0 . The objective is then to have robust and exact estimation of $f_{0}, \dot{f}_{0} \ldots f_{0}^{(n)}$. The following scheme offers such an estimate:

$$
\left\{\begin{array}{l}
\dot{z}_{0}=-\lambda_{n} L^{\frac{1}{n+1}}\left|z_{0}-f(t)\right|^{\frac{n}{n+1}} \operatorname{sign}\left(z_{0}-f(t)\right)+z_{1} \\
\dot{z}_{1}=-\lambda_{n-1} L^{\frac{1}{n}}\left|z_{0}-f(t)\right|^{\frac{n-1}{n}} \operatorname{sign}\left(z_{0}-f(t)\right)+z_{2} \\
\cdots \\
\dot{z}_{n-1}=-\lambda_{1} L^{\frac{1}{2}}\left|z_{0}-f(t)\right|^{\frac{1}{2}} \operatorname{sign}\left(z_{0}-f(t)\right)+z_{n} \\
\dot{z}_{n}=-\lambda_{0} L\left|z_{0}-f(t)\right|
\end{array}\right.
$$

where $\lambda_{i}$ are tuning parameters for $i=1, \ldots, n$. Although an infinite sequence $\lambda_{i}$ can be built, it has been shown that $\left\{\lambda_{0}, \lambda_{1}\right\}=\{1.1,1.5\}$ suffice for the zero- and first-order derivatives.

According to [14], if the input noise satisfy $|w| \leq \epsilon$ for all $t \geq 0$, then the differentiation ensured accuracy satisfies the following inequality:
Theorem 1. Let the input noise satisfy $|w(t)| \leq \epsilon$ for all $t \geq 0$. Then the following inequalities are established in finite-time $T>0$, for some positive constant $\rho_{i}$ depending exclusively on the parameters $\lambda_{1}, \ldots, \lambda_{n}$ of the differentiator:

$$
\left|z_{i}(t)-f_{0}^{(i)}(t)\right| \leq \rho_{i} L^{\frac{1}{n+1}} \epsilon^{\frac{n-i+1}{n+1}}, \forall t \geq T, i=0,1, \ldots, n
$$

Also, all solutions of this scheme are Lyapunov stable. For proofs, the reader is invited to refer to [14].

\section{B. First order sliding mode control}

Here, we present a brief introduction to first-order sliding mode control. Consider the following non-linear system:

$$
\dot{x}(t)=f(t, x, u, d)
$$

where $x \in \mathbb{R}^{n}$ is the state vector, $u \in \mathbb{R}^{m}$ is the control input vector and $d \in \mathbb{R}^{n}$ is a vector containing perturbations and possibly uncertainties within the system. Then, consider the following surface in the state space:

$$
\mathcal{S}=\left\{x \in \mathbb{R}^{n}, \sigma(x)=0\right\}
$$

where $\sigma: \mathbb{R}^{n} \rightarrow \mathbb{R}$ is a continuously differentiable function.

The intention is to design $\sigma(x)$ in such a way that system (5), under feedback control, behaves with prescribed performance. In this sense, if the control input is selected guaranteeing $\dot{\sigma}(x)=-\eta \operatorname{sign}(\sigma(x))$, where $\eta>0$, one can consider a candidate Lyapunov function as

$$
V=\frac{1}{2} \sigma^{2}
$$

and, computing its time derivative, we have that

$$
\begin{aligned}
\dot{V} & =\sigma \dot{\sigma} \\
& =-\eta|\sigma(x)|
\end{aligned}
$$

which implies $\dot{V} \leq 0$, hence the origin of $\sigma(x)$ is globally stable. Moreover, it is clear that $|\sigma|=\sqrt{2} V^{0.5}$ and, consequently, it implies that

$$
V^{0.5}(t)=V^{0.5}(0)-\sqrt{2} \eta t
$$

meaning that, for $\sigma(0) \neq 0$, solution of (7) becomes zero in a finite time [15].

Here, we can discriminate two interesting phases of this methodology: the reaching phase, which is guaranteed if condition (7) is satisfied and describes the motion of the system towards the surface $\sigma(x)$, and the sliding phase, describing the motion of the system in the prescribed surface.

A direct consequence of the establishment of a sliding motion is the equivalent control [16], which is the control action required to keep the trajectory on the sliding surface. 


\section{Design of the Control Architecture}

Our control strategy has two phases. First, using the control $S_{i n}$ we are going to shift the substrate to a positive value ensuring the system controllability, and in parallel to stabilize $x_{1}$ at a desired position $x_{1}^{d}>0$ applying the control $D$. Once these goals are reached, we will commutate the control goal for $S_{i n}$ to stabilization of $x_{2}$ at its desired position $x_{2}^{d}$. Below all these control laws are described in details, and their properties are substantiated.

This commutation is performed by a supervisor scheme, whose logic is given by:

- Stabilization of $x_{1}$ is always activated;

- The first switching law is to be activated for $t<t_{\text {switch }}$;

- The second switching law is to be activated for $t \geq$ $t_{\text {switch }}$;

where $t_{\text {switch }}$ is the first instant of time in which $\left|x_{1}-x_{1}^{d}\right|=$ 0 .

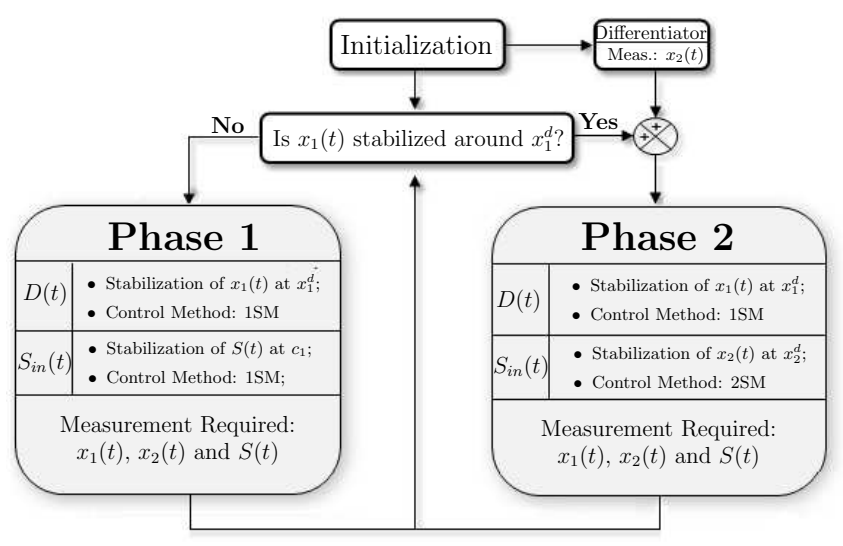

Fig. 1. Overview of the Control Architecture

\section{A. Stabilization of $x_{1}$}

Consider the following surface:

$$
\sigma_{1}\left(x_{1}\right)=x_{1}-x_{1}^{d}
$$

where $x_{1}^{d} \neq 0$ is an arbitrary point for $x_{1}$ to be stabilized.

As we can see from equation (1), if the concentration $x_{i}$ is in a steady-state position, i.e., $\dot{x}_{i}(t)=0$ for $t \geq 0$, then there are two possibilities: either $x_{i}=0$ or $D(t)=\mu_{i}(S(t))$. With the latter case in mind, we can state the following result:

Theorem 2. Consider model (1) and surface (8). If the dilution rate is selected such as

$$
D= \begin{cases}\overline{\mu_{1}}(S)+\chi & \text { if } \sigma_{1} \geq 0 \\ \underline{\mu_{1}}(S)-\chi & \text { if } \sigma_{1}<0\end{cases}
$$

where $\chi \in\left(0, \mu_{1}(S(0))\right)$ is a tuning parameter, $\mu_{1}(S)=$ $\min _{a_{i} \in A_{1}, b_{i} \in B_{1}} \bar{\mu}(S)$ and $\overline{\mu_{1}}=\max _{a_{i} \in A_{1}, b_{i} \in B_{1}} \overline{\mu(S)}$ then the closed-loop system is globally finite-time stable with respect to the output $\sigma_{1}$, provided that $S(t)>S(0) \quad \forall t \geq 0$.

Proof. Consider the following candidate Lyapunov function for (8), which is proper and positive definite with respect to $\sigma_{1}:$

$$
V_{1}=\frac{1}{2} \sigma_{1}^{2}
$$

whose time derivative is given by

$$
\begin{aligned}
\dot{V}_{1} & =\sigma_{1} \dot{\sigma} \\
& =\sigma_{1}\left(\mu_{1}(S)-D\right) x_{1}
\end{aligned}
$$

and hence, choosing $D$ as given by (9) and noticing that due to the form of $\sigma_{1}$ and that $x_{1}(0) \neq 0$, one has $\dot{V}_{1}<0$ for any $x_{1}^{d} \neq 0$, proving the claim.

Clearly, the given control law for $D$ uses information about of $x_{1}$ and also $S$, and the latter has to be separated from zero (bigger than $S(0)>0$ to have $D(t)>0$ for the selected value of $\chi$ ). Hence, it is necessary to design a control law for $S$, which can be done using $S_{i n}$.

In this sense, as it will be a key point in this development, let us introduce the following assumption:

Assumption 1. Defining $\xi(S)=\mu_{1}(S)-\mu_{2}(S)$, for any $a_{i} \in A_{i}$ and $b_{i} \in B_{i}$, let there exist a constant $S_{a_{i}, b_{i}}$ such that $\xi\left(S_{a_{i}, b_{i}}\right)=0$ and $\xi(S)>0$ for $S>S_{a_{i}, b_{i}}$.

Assumption 2. For any $a_{i} \in A_{i}$ and $b_{i} \in B_{i}$, the equality $\frac{\partial \xi\left(S_{i}\right)}{\partial S}=0$ is satisfied for some $S_{i}<S_{a_{i}, b_{i}}$.

Remark 1. Assumptions 1-2 are fulfilled, for instance, with a sufficient (but not necessary) condition $a_{1}>\overline{a_{2}}$ and $\overline{b_{1}}<$ $\underline{b_{2}}$.

\section{B. First Switching Law}

This first structure is to be activated for $t<t_{\text {switch }}$. The objective here is to keep $S$ away from zero, since it would cause $D=0$ (since $\left.\mu_{1}(0)=\mu_{2}(0)=0\right)$ and we will have no control action on $x_{1}$.

In order to design a control law for $S_{i n}$, it is necessary to note that the point $S_{i}$, defined in Assumption 2, corresponds to the value of $S$ at which the system looses its controllability for the variable $x_{2}$ with the control $S_{i n}$. In addition, the system is not controllable at all, if $S=0$. Then the goal is to shift $S$ out of the dangerous region. To this end, select a constant $c_{1} \notin\left[\underline{S_{i}}, \overline{S_{i}}\right]$, where

$$
\begin{aligned}
& \underline{S}=\min _{\substack{a_{i} \in A_{i} \\
b_{i} \in B_{i}}} S_{i} \\
& \bar{S}=\max _{\substack{a_{i} \in A_{i} \\
b_{i} \in B_{i}}} S_{i}
\end{aligned}
$$

thus stabilization of $S$ at $c_{1}$ will ensure that at least in the instant of commutation to regulate $x_{2}$, the latter is controllable.

Hence, we can state the following result:

Theorem 3. Consider model (1). By means of a switching law given by

$$
S_{\text {in }}=D^{-1} \begin{cases}S_{\max } & \text { if } S<c_{1} \\ 0 & \text { if } S \geq c_{1}\end{cases}
$$


where

$$
S_{\max }=D S+\sum_{i=1}^{2} k_{i} x_{i} \max _{a_{i} \in A_{i}, b_{i} \in B_{i}} \mu_{i}(S)+\epsilon
$$

with $\epsilon>0$, the output $S-c_{1}$ is globally finite-time stabilized for (1), provided that $D(t)>0$, for all $t \geq 0$.

Proof. Consider the following candidate Lyapunov function:

$$
V_{S}=\frac{1}{2}\left(S-c_{1}\right)^{2}
$$

which has time derivative given by

$$
\dot{V}_{S}=\left(S-c_{1}\right) \dot{S}
$$

and note that with the selected commutation law, one has $\dot{V}_{S}<0 \forall S \neq c_{1}$ implying the stated result.

Also, note that with the controls (9) and (10), if $S(0)>0$, then $D(t)>0$ and $S(t)>0$ for all $t \geq 0$.

\section{Second Switching Law}

This second structure is to be active once $x_{1}$ is stabilized in surface (8), i.e., for $t>t_{\text {switch }}$. The objective here is then to stabilize the remaining species $x_{2}$ at an arbitrary point $x_{2}^{d}>0$, which can be done by means of $S_{i n}$.

Remark 2. Due to the discontinuous control law (9), an immediate consequence is the establishment of sliding motion on the surface $\sigma_{1}=0$, meaning that the control $D(t)$ can be considered in its equivalent form [16] $D_{e q}(t)=\mu_{1}(S(t))$, which is assumed in this subsection.

As it can be seen from model (1), if concentration $x_{2}$ is in steady-state, i.e., $\dot{x}_{2}=0$, the equivalent control on $D(t)$ implies that stabilization can be achieved if $\xi(S)=0$, i.e., $S=S_{a_{i}, b_{i}}$.

Clearly, if all parameters of the kinetic rates (2) were perfectly known, then the intersection point $S_{a_{i}, b_{i}}$ would be readily available and the stabilization is easily solved. However, as aforementioned, these parameters are uncertain and therefore the control law designed in this section must provide robust stabilization of $x_{2}$.

In this sense, define an auxiliary variable $y=x_{2}-x_{2}^{d}$ and the surface

$$
\sigma_{2}(y)=\kappa y+\dot{y}
$$

where $\kappa>0$ is a tuning parameter.

To calculate the value of $\sigma_{2}$ we need the information about $\dot{y}$ (or $\dot{x}_{2}$ ), which is not measurable, then, the presented differentiator scheme (3) can be used to compute $\dot{y}$ as follows:

$$
\begin{aligned}
& \dot{z}_{0}=-1.5 L^{\frac{1}{2}}\left|z_{0}-y(t)\right|^{\frac{1}{2}} \operatorname{sign}\left(z_{0}-y(t)\right)+z_{1} \\
& \dot{z}_{1}=-1.1 L\left|z_{0}-y(t)\right|
\end{aligned}
$$

thus we have that $z_{1} \rightarrow \dot{y}$ in a finite-time in the noisefree case, having $L>0$ as another tuning parameter for the scheme (it can be estimated for a given set of values of $S, x_{1}$ and $x_{2}$ ). Note that this differentiator can be launched during the first phase, and its convergence time can be adjusted to ensure that when $\sigma_{1}$ is stabilized, the derivative estimates are also converged.

In this light, let us state the following result:

Theorem 4. Consider model (1) under assumptions 1-2 and surface (11). Considering the following switching law

$$
S_{i n}= \begin{cases}\frac{\alpha}{\beta D}, & \text { if } \quad \sigma_{2} \geq 0 \\ 0, & \text { if } \quad \sigma_{2}<0\end{cases}
$$

where

$$
\begin{aligned}
\alpha & =\max _{a_{i} \in A_{i}, b_{i} \in B_{i}} \frac{\partial \xi}{\partial S}\left(D S+\sum_{i=1}^{2} k_{i} x_{i} \mu_{i}(S)\right)+\xi^{2}(S)+\chi \\
\beta & =\min _{a_{i} \in A_{i}, b_{i} \in B_{i}} \frac{\partial \xi}{\partial S}
\end{aligned}
$$

with $\chi>0$ as a tuning parameter. Then, the closed-loop system is stable with respect to the output $\sigma_{2}$ in a certain domain, provided that $S(t)>S_{i}$ for all $t \geq 0$.

Proof. Consider the candidate Lyapunov function:

$$
V_{2}=\frac{1}{2} \sigma_{2}^{2}
$$

whose time derivative, recalling that $D(t)$ is in equivalent control mode (see Remark 2), is given by

$$
\begin{aligned}
\dot{V}_{2} & =\sigma_{2} \dot{\sigma}_{2} \\
& =\sigma_{2}(\kappa \dot{y}+\ddot{y}) \\
& =\sigma_{2}\left(-\frac{\partial \xi}{\partial S} \dot{S} x_{2}+\xi^{2}(S) x_{2}-\kappa \xi(S) x_{2}\right)
\end{aligned}
$$

where, reorganizing in a way that input $S_{i n}$ appears explicitly, we have

$$
\begin{aligned}
\dot{V}_{2} & =\sigma_{2} x_{2}\left(-\frac{\partial \xi}{\partial S} D S_{i n}+\frac{\partial \xi}{\partial S}\left(D S+\sum_{i=1}^{2} k_{i} x_{i} \mu_{i}(S)\right)\right. \\
& \left.+\xi^{2}(S)-\kappa \xi(S)\right)
\end{aligned}
$$

and thus, by means of control laws (13), one has that $\dot{V} \leq 0$, proving the claim.

Remark 3. As clearly stated on subsection III-A, the differentiator (3) requires the input signal to be Lipschitz. However, once $x_{2}(t)$ attains the sliding motion, the signal $y(t)$ in (11) becomes discontinuous. As information on $\dot{y}(t)$ is also required, the issue of differentiating such a signal might be alleviated by means of a low-pass filter.

Remark 4. Due to space limitations, time-varying fluctuations on parameters $a_{i}$ and $b_{i}$ will not be discussed in this work. However, it can be tackled in a very natural manner by the methodology presented. Indeed, the influence of these fluctuations in the control law would appear in (14) (for instance, through terms as $\left.\frac{d a_{i}}{d t}\right)$ and, if this variations are slow (which is reasonable for biochemical processes), it can be simply considered as a perturbation. 
TABLE I

RANDOM PARAMETER SETS

\begin{tabular}{|c|c|c|}
\hline & {$\left[a_{1}, b_{1}\right]$} & {$\left[a_{2}, b_{2}\right]$} \\
\hline Parameter Set 1 & {$[1.9867,5.9067]$} & {$[1.5100,3.0333]$} \\
\hline Parameter Set 2 & {$[2.0222,6.0933]$} & {$[1.5300,2.9800]$} \\
\hline Parameter Set 3 & {$[2.0133,5.8800]$} & {$[1.4767,3.0333]$} \\
\hline Parameter Set 4 & {$[2.000,6.000]$} & {$[1.5000,3.0000]$} \\
\hline
\end{tabular}

\section{NUMERICAL EXAMPLE}

In this section, we present a numerical example in order to verify the usefulness of the developed methodology. Consider model (1) with initial conditions $S(0)=7$ and $x_{i}(0)=1.5$. Moreover, we consider the control inputs to be constrained such as $D \in[0,2]$ and $S_{i n}=[0,15]$. It is assumed that the parameter uncertainties satisfy the following intervals:

$$
\begin{array}{ll}
A_{1}=[1.90,2.04], & A_{2}=[1.47,1.53] \\
B_{1}=[5.70,6.12], & B_{2}=[2.85,3.06]
\end{array}
$$

and, for simulation purposes (i.e., the controller has knowledge only of the intervals $A_{i}$ and $B_{i}$ above), let us consider different parameter sets of the nominal model, as given in Table I. Also, for simplicity, we consider $k_{1}=k_{2}=1$.

Following the first design step, we selected the stabilization point for $x_{1}$ as $x_{1}^{d}=3$ and proceed as described in subsection IV-A. For the first switching law, the considered uncertainties suggest that $[\underline{S}, \bar{S}]=[3.75,10.7]$, hence we arbitrarily select $c_{1}=3.6$. The control law is then given as presented in subsection IV-B.

Regarding the second switching law, we select $x_{2}^{d}=1$ and compute $y(t)$ while, selecting the Lipschitz constant as $L=$ 50 on the differentiator (3), we estimate $\dot{y}(t)$. The control law is then given as presented in subsection IV-C.

Also, as discussed in Remark 3, we will consider a lowpass filter to applied on $y(t)$ as soon as $x_{2}(t)$ attains slidingmotion on surface (11). This filter is given by the following transfer function:

$$
\mathcal{H}(s)=\frac{1}{0.1 s+1}
$$

As result of the simulation of all the scenarios indicated by Table I, Fig. 2 show the time evolution of the error for both species. It can be readily seen that, despite of the variation in all parameters, the stabilization problem is still solved for both species. However, it can be clearly seen that the stabilization of $x_{2}(t)$ is more sensitive to this variation.

Now, for sake of visualization, let us consider only the parameter set 4 from Table I. In this sense, Fig. 3 shows more comprehensively the results obtained, where one can see the stabilization of the concentration of both species at the desired levels. Also, as it is an important part of the control architecture, one can readily see the stabilization of $S(t)$ at $c_{1}$ until the second control phase (i.e., aiming stabilization of $\left.x_{2}(t)\right)$ takes place. In Fig. 4, the control inputs $D$ and $S_{i n}$, issued from this simulation scenario, are illustrated.
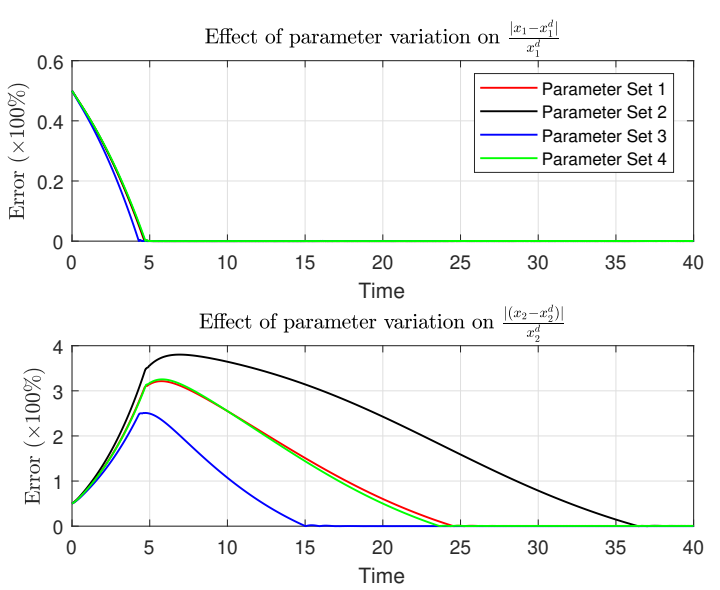

Fig. 2. Error evolution for the stabilization of $x_{1}(t)$ (above) and $x_{2}(t)$ (below)

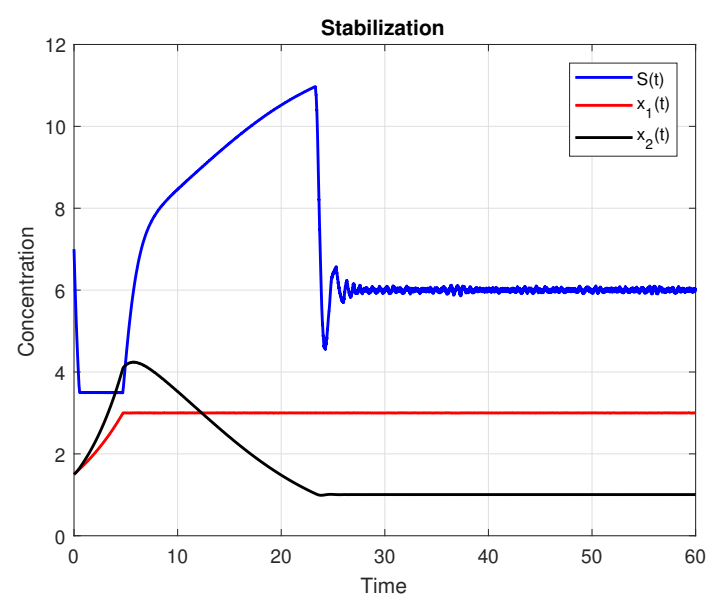

Fig. 3. Stabilization of $S$ and $x_{i}$ (Parameter set 4)

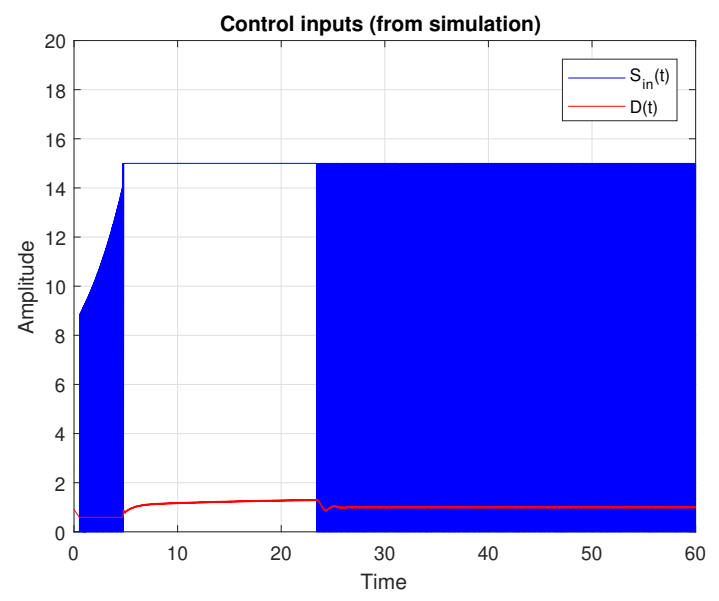

Fig. 4. Control inputs $D$ and $S_{\text {in }}$ (Parameter set 4) 


\section{Conclusions And Perspectives}

In this work, we further discussed the problem of stabilization of two species inside a bioreactor, under competition for a single limiting substrate. Considering all parameters on the kinetic rates to be uncertain for the values within a certain given interval, we developed discontinuous control laws that insure the persistence of all species. These control laws have been designed in two different architectures, which are switched under certain conditions by a supervisor. As it was shown, the concentration of species $x_{1}$ can be globally stabilized at an arbitrary point, while the concentration of species $x_{2}$ can be stabilized only locally.

As a topic of future works, it would be interesting to better describe the domain of attraction obtained for the local stabilization performed by the second switching law - which might not be a trivial task since it deals with a hybrid system. Another interesting direction might be pursued by dropping the harsh assumption of full state measurement, implying that the study of observer-based control might be carried out aside the methodology presented here.

Also, robustness against time-varying parameters can be tackled and sensitivity analysis can be an interesting option to better characterize the effect of the considered uncertainties on the closed-loop system and its performance.

Regarding future practical implementations, a study aiming chattering reduction and consistent discretization [17] of the sliding mode controllers might be carried out.

\section{REFERENCES}

[1] J. Singh, N. Kaushik, and S. Biswas, "Bioreactors technology \& design analysis," The Scitech Journal, pp. 2347-7318, 2014.

[2] H. L. Smith and P. Waltman, The theory of the Chemostat. Cambridge University Press, 1995.

[3] P. Leenheer and H. Smith, "Feedback control for chemostat models," Journal of Mathematical Biology, vol. 46, pp. 48-70, 2003.

[4] F. Mazenc, M. Malisoff, and J. Harmand, "Further results on stabilization of periodic trajectories for a chemostat with two species," IEEE Transactions on Automatic Control, vol. 53, no. Spécial issue, pp. 66-74, 2008. [Online]. Available: https://hal.inria.fr/hal-00857806

[5] D. Dochain and G. Bastin, On-line Estimation and Adaptive Control of Bioreactors, ser. Process Measurement and Control, , Ed. Elsevier, 1990, no. 1.

[6] G. Robledo, "Control of the chemostat: Some Results," Theses, Université Nice Sophia Antipolis, Mar. 2006. [Online]. Available: https://tel.archives-ouvertes.fr/tel-00069800

[7] C. Kravaris and G. Savoglidis, "Tracking the singular arc of a continuous bioreactor using sliding mode control," Journal of the Franklin Institute, vol. 349, pp. 1583-1601, 2011.

[8] F. Mairet and O. Bernard, "Robustness of Closed-Loop Control to Biodiversity: a Didactic Example," in 19th IFAC World Congress, Cape Town, South Africa, Aug. 2014, pp. 5321-5326. [Online]. Available: https://hal.inria.fr/hal-01094693

[9] J. Gouzé and G. Robledo, "Robust control for an uncertain chemostat model," International Journal of Robust and Nonlinear Control, vol. 16, no. 3, pp. 133-155, 2006.

[10] D. Dochain, Ed., Automatic Control of Bioprocesses, ser. Control systems, Robotics and Manufacturing Series. ISTE Ltd., 2008.

[11] W. Perruquetti and J. Barbot, Eds., Sliding Mode Control in Engineering, ser. Control Engineering Series. Marcel Dekker, 2002.

[12] F. Montefusco, O. E. Akman, O. S. Soyer, and D. G. Bates, "Ultrasensitive negative feedback control - a natural approach for the design of syntetic controllers," PLoS One, vol. 11, no. 8, 2016.

[13] International Journal of Control, vol. 91. Taylor and Francis, 2018, number 9: special issue on differentiators.
[14] A. Levant, "Higher-order sliding modes, differentiation and outputfeedback control," International Journal of Control, vol. 79, pp. 924941, 2003.

[15] Y. Shtessel, C. Edwards, L. Fridman, and A. Levant, Sliding Mode Control and Observation, ser. Control Engineering. Birkhauser, 2010.

[16] V. I. Utkin, Sliding Modes in Control and Optimization, ser. Communications and Control Engineering. Springer, 1992.

[17] A. Polyakov, D. Efimov, and B. Brogliato, "Consistent Discretization of Finite-time and Fixed-time Stable Systems," SIAM Journal on Control and Optimization, vol. 57, no. 1, pp. 78-103, 2019. [Online]. Available: https://hal.inria.fr/hal-01838712 